\title{
Extensive study of the electron donor 1,1,4,4-tetrathiabutadiene (TTB) and of its charge transfer crystal with TCNQ
}

\author{
Nicola Castagnetti ${ }^{\mathrm{a}}$, Alberto Girlando ${ }^{\mathrm{a}, *}$, Matteo Masino ${ }^{\mathrm{a}}$, Corrado Rizzoli ${ }^{\mathrm{a}}$, \\ M. R. Ajayakumar ${ }^{\mathrm{b}}$, Marta Mas-Torrent ${ }^{\mathrm{b}}$, Concepció Rovira ${ }^{\mathrm{b}}$ \\ ${ }^{a}$ Dipartimento di Scienze Chimiche, della Vita e della Sostenibilità Ambientale, and INSTM-UdR Parma, Università \\ di Parma, Parco Area delle Scienze 17/a, I-43124 Parma, Italy \\ ${ }^{\mathrm{b}}$ Department of Molecular Nanoscience and Organic Materials, Institut de Ciència de Materials de Barcelona (ICMAB- \\ CSIC) and Networking Research Center on Bioengineering, Biomaterials and Nanomedicine (CIBER-BBN), ES-08193 \\ Bellaterra, Spain
}

\begin{abstract}
In the spirit of the renewed interest in mixed stack charge-transfer (CT) crystals, made up by alternating $\pi$ electron-donor and acceptor molecules, we focus attention on a forgotten donor, 1,1,4,4tetrathiabutadiene (TTB), synthesized more than 35 years ago. We present a spectroscopic and computational characterization of the neutral TTB, and fully characterize the CT crystal with TCNQ. TTB-TCNQ is a mixed stack crystal, with limited degree of CT (about 0.1), despite TTB electron donating strength is only a little smaller than that of the famous TTF. This finding is explained by the small value of the Madelung energy, that we evaluate by a well tested computational approach.
\end{abstract}

Keywords:

$\pi$-electron donor

Mixed stack charge transfer

crystals Organic semiconductors

DFT

*Corresponding author.

E-mail address: alberto.girlando@unipr.it

\section{Introduction}

Interest in mixed stack organic charge transfer (CT) crystal has been recently renovated by their potential application as ambipolar semiconductors [1] or as ferroelectrics [2]. In this context, there has been a flourishing of papers aimed at characterizing different combinations of electron donor (D) and acceptor (A) molecules [3-8], in order to acquire knowledge on the structure-properties relations, and eventually gain control on the packing design, the band gap, and the degree of charge transfer ( $\rho$ ) of these systems. Besides synthesizing new D and A molecules, there has been a rediscover of old systems, e.g., DBTTF-TCNQ $[9,10]$, investigated through modern methods. Following the latter approach, we found an interesting electron donor, 1,1,4,4-tetrathiabutadiene (TTB, Scheme 1), for which just the 
synthesis and a preliminary characterization of some of its CT crystals is present in the literature [11].

Here we report a detailed spectroscopic, structural and computational characterization of TTB and of the corresponding co-crystal TTB-TCNQ, extending the knowledge basis of mixed stack CT crystals, and showing that this forgotten molecule and its variants have the potential of affording new systems with perspective applications.

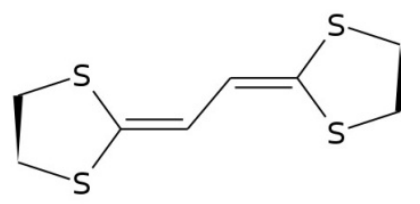

Scheme 1: TTB

\section{Experimental Methods}

\section{Sample preparation}

The donor TTB was synthesized following the procedure sketched in Ref. [11], that is by coupling of ethanedithiol and dimethoxydihydrofuran in the presence of $\mathrm{BF}_{3}$ and subsequent oxidation of the resulting compound. Complete synthetic procedure and characterization is reported in the Supporting Information. Crystals of TTB-TCNQ several mm long were obtained by the slow diffusion at room

temperature of two saturated acetonitrile solutions of the components. Millimeter size crystals could be extracted after five days.

\section{$X$-ray diffraction measurements}

A single crystal of TTB-TCNQ was mounted on a Bruker SMART 1000 CCD diffractometer equipped with graphite monochromated Mo K $\alpha$ radiation $(\lambda=0.71073 \AA)$ fine-focus sealed tube. The intensity data were collected using $\omega$ scan at 295(2) K. Cell refinements and data reductions were performed using the Bruker SAINT software [12]. The structures were solved by direct methods using the program SIR97 [13] and refined with full-matrix least-squares based on $F^{2}$ using the program SHELXL2014/7 [14]. All non-hydrogen atoms were refined anisotropically. Hydrogen atoms were placed geometrically and refined using a riding model approximation, with $\mathrm{C}-\mathrm{H}=0.93-0.97 \AA$ and with $U_{\text {iso }}(\mathrm{H})=1.2 U_{\text {eq }}(\mathrm{C})$. The molecular graphics were prepared using Mercury [15] and Jmol [16] programs. 
Infrared (IR) spectra of the crystals were recorded with a Bruker IFS66 Fourier transform IR (FT-IR) spectrometer coupled to an IR microscope Hyperion 1000. Spectral resolution: $2 \mathrm{~cm}^{-1}$. The Raman spectra were recorded with a Renishaw 1000 Raman spectrometer equipped with the appropriate edge filter, and coupled to a Leica M microscope. A Lexel Kr laser was used as light source. Exciting line: $676 \mathrm{~nm}$.

\section{Computational Methods}

The vibrational frequencies of TTB and $\mathrm{TTB}^{+}$have been calculated with the GAMESS package [17], using Density Functional (DFT) with unrestricted B3LYP and the 6-31G(d) basis set. This combination is known to satisfactorily reproduce the molecular vibrations of relatively large organic molecules,

provided tight geometry optimization and fine grids are used in the DFT analysis [18]. To calculate the CT integral of TTB-TCNQ we have followed the dimer approach [19,20], using the Gaussian09 package [21] to compute singlet and triplet ground states for a DA dimer. The basis set was again 6$31 G(d)$, whereas we chose the unrestricted $\omega B$ 97XD functional, which is more suitable for modeling intermolecular CT. The average charge residing on TTB and TCNQ molecules has been estimated on the basis of the computed Hirshfeld atomic charges [21]. The electrostatic interaction $V$ within a DA pair and the Madelung energy $M$ have been calculated by adopting the point-charge approximation of the molecular charge density based on ESP atomic charges . The crystal electrostatic sums have been obtained for finite clusters of increasing size using the MESCAL code [22].

\section{Results and Discussion}

\subsection{TTB}

The minimum energy conformation of neutral and ionic TTB in gas phase has $C_{\mathrm{i}}$ symmetry, with a planar central skeleton and out-of-plane staggered arrangement of the terminal $\mathrm{CH}_{2}-\mathrm{CH}_{2}$ groups. As chemical intuition suggest, the HOMO is concentrated on the double bonds and sulfur atoms (Figure 1). The former will then be weakened upon electron removal, as it happens with electron Donors based on the tetrathiafulvalene (TTF) skeleton. 


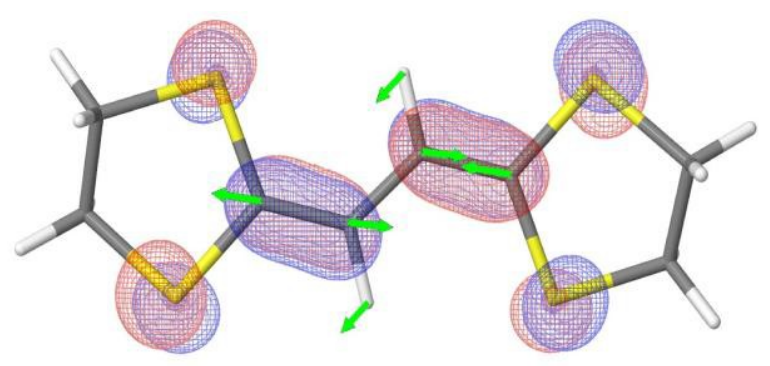

Fig. 1. Representation of the TTB HOMO. The eigenvectors of the IR active antisymmetric $\mathrm{C}=\mathrm{C}$ stretching vibration are also shown (green arrows).

The HOMO energy can be taken as a relative measure of the ionization potential. The TTB HOMO energy is calculated to be $-4.59 \mathrm{eV}$, only $0.13 \mathrm{eV}$ lower than that of TTF $(-4.46 \mathrm{eV}$, calculated by the same method). Then apparently the electron donor strength of TTB is only slightly smaller than that of TTF, in agreement with measured UPS vertical-ionization energy, less with the relevant ionization potentials [11].

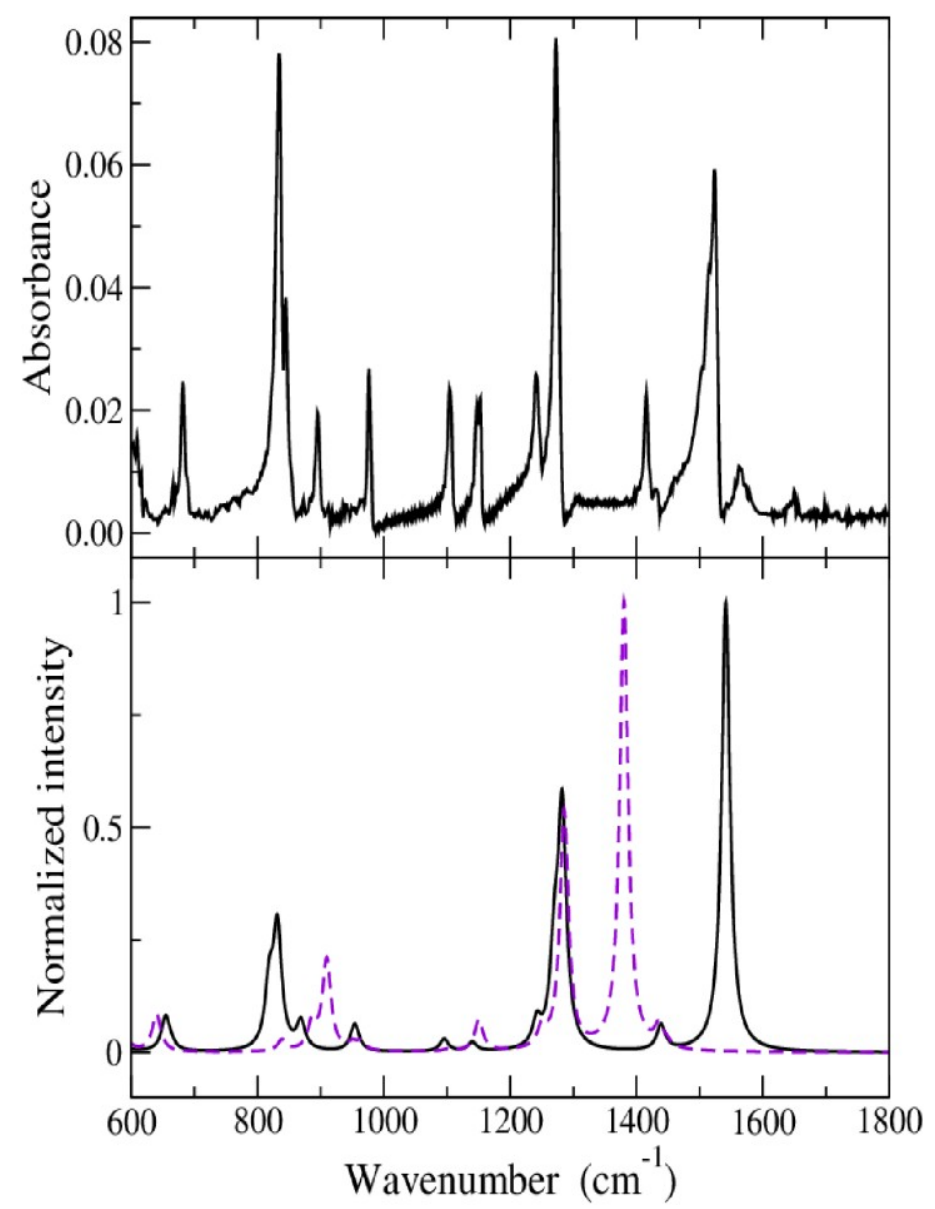

Fig. 2. Top panel: Experimental IR spectrum of TTB powders. Bottom panel: Calculated IR spectrum of neutral TTB (black line) and $\mathrm{TTB}^{+}$(violet dashed line). 
For the free TTB molecule in the $C_{i}$ conformation, group theory predicts 30 vibrational modes of $a_{g}$ symmetry, active in Raman, and 30 modes of $a_{u}$ symmetry, infrared (IR) active. Calculations yield the vibrational assignment of the neutral molecule, as reported in the Supporting Information. The

experimental IR and computed spectra are compared in Fig. 2. The calculated IR spectrum of $\mathrm{TTB}^{+}$is also reported in the same Figure, in order to put in evidence the charge sensitive vibrational modes which allow the estimation of the degree of charge transfer $[23,24]$ in the CT complexes in which TTB may be involved. It is seen that the antisymmetric $\mathrm{C}=\mathrm{C}$ stretching, whose eigenvectors are pictorially represented in Fig. 1, and occurring at $1524 \mathrm{~cm}^{-1}$, is the one exhibiting the largest ionization frequency shift, about $160 \mathrm{~cm}^{-1}$. Of course, also the corresponding Raman active $\mathrm{C}=\mathrm{C}$ symmetric stretching (Table S1) has a large ionization shift, but this mode mixes with the symmetric stretching of the central C-C bond, and in any case the totally symmetric modes, being coupled with the CT electron, cannot be used to estimate the ionicity of mixed stack CT crystals [25]. We finally notice that the $a_{u}$ C-S stretching mode located at $834 \mathrm{~cm}^{-1}$ is expected to exhibit a considerable (about $70 \mathrm{~cm}^{-1}$ ) upward frequency shift upon ionization (Fig. 2 and Table S1).

\subsection{TTB-TCNQ}

TTB-TCNQ crystallizes in the triclinic system, space group P-1, with one DA pair per unit cell. The basic crystallographic parameters are reported in Table 1. Full information is given in the deposited CIF files (CCDC: 1576020). The present structure is different from the monoclinic one reported in Ref. [11], probably a case of polymorphism due to a different crystallization procedure.

Table 1. Room temperature crystal structure of TTB-TCNQ

\begin{tabular}{ll}
\hline Space group & $P-1$ \\
$Z$ & 1 \\
$T / \mathrm{K}$ & $294(2)$ \\
$a / \AA$ & $6.9177(7)$ \\
$b / \AA$ & $8.9769(9)$ \\
$c / \AA$ & $9.0882(9)$ \\
$\alpha(\mathrm{deg})$ & $68.2461(15)$ \\
$\beta(\mathrm{deg})$ & $77.9771(17)$ \\
$\gamma(\mathrm{deg})$ & $88.7500(18)$ \\
$V / \AA^{3}$ & 511.728 \\
$\mathrm{R}, \mathrm{wR} 2$ factor & $4.26,0.109$ \\
\hline
\end{tabular}




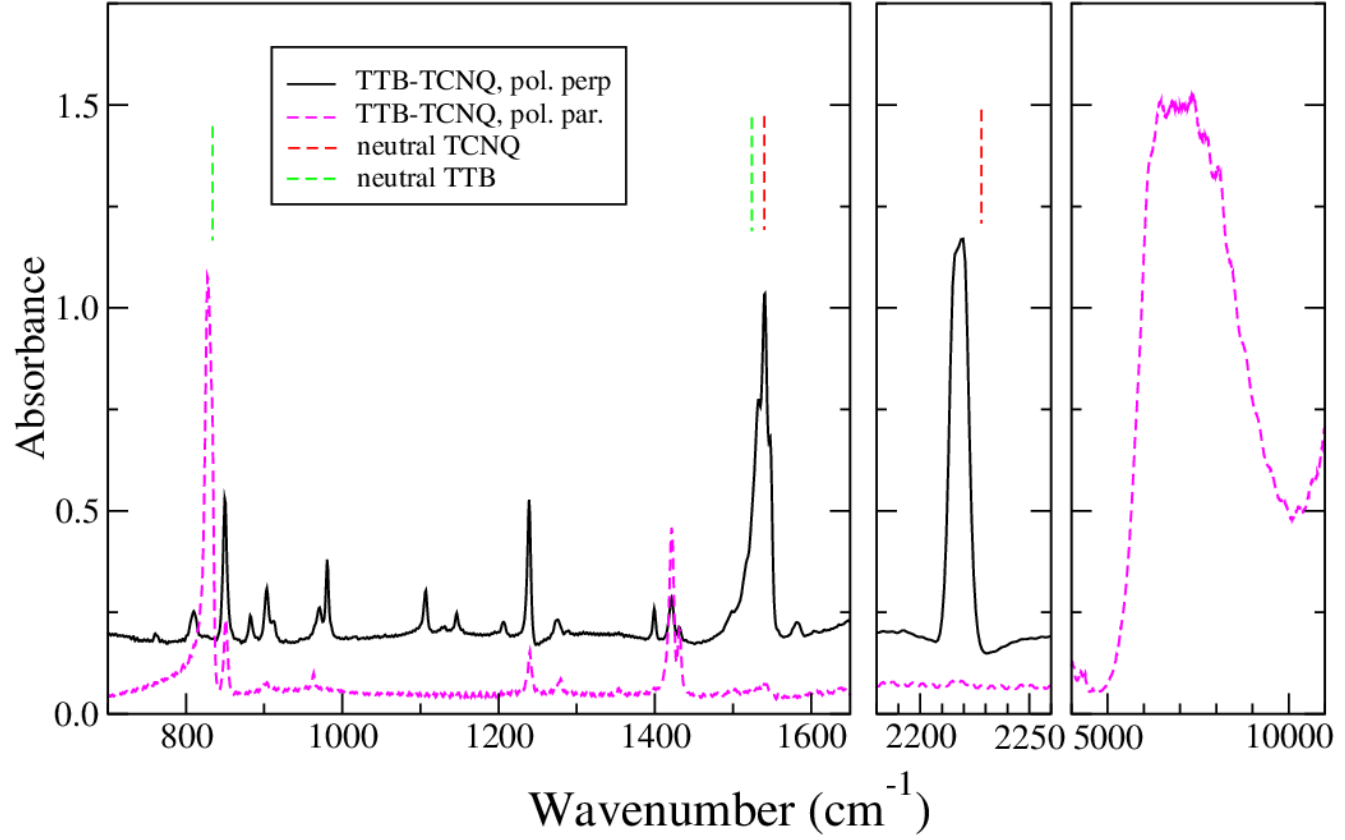

Fig. 4. Polarized IR spectra of TTB-TCNQ in the spectral regions of the charge sensitive vibrations and of the CT transition. Black continuous line: electric vector perpendicular to the stack axis. Magenta dashed line: electric vector parallel to the stack axis. The spectra are offset for clarity. Red and green dashed lines indicate the frequency of the charge sensitive vibrations in neutral TCNQ and TTB, respectively.

frequency, occurring at $2218 \mathrm{~cm}^{-1}$, indicates $\rho=0.2$, but the $\mathrm{CN}$ stretching frequency is known to

give unreliable, generally overestimated, $\rho$ values [26]. On the other hand, the TCNQ $b_{1 u} v_{20} C=C$ antisymmetric stretching is very close to the analogous vibration of TTB $a_{u} v_{36}$ : In neutral TCNQ and TTB they occur at 1545 [27] and at $1524 \mathrm{~cm}^{-1}$ (Table S1), respectively. In this spectral region TTBTCNQ exhibits an absorption with a complex structure, with several peaks. If we attribute the main peak, at $1540 \mathrm{~cm}^{-1}$, to the TCNQ vibration, we find $\rho=0.1$, but the frequency of the TTB mode is not safely identified. Finally, the C-S antisymmetric stretch of TTB is located at $850 \mathrm{~cm}^{-1}$, slightly upward in respect to the neutral molecule, as expected, but its precise calibration and actual ionization frequency shift are presently not known. The Raman spectra, shown in Fig. 5, confirms that TTBTCNQ is well on the neutral side, as the observed frequencies are very close to the ones of the neutral molecules - the effect of the interaction of the totally symmetric modes with the CT transition is small when $\rho$ is close to 0 or 1 [25]. To summarize, from vibrational spectra we estimate the degree of CT of TTB-TCBQ around 0.1, with a rather large uncertainty $( \pm 0.06)$. 


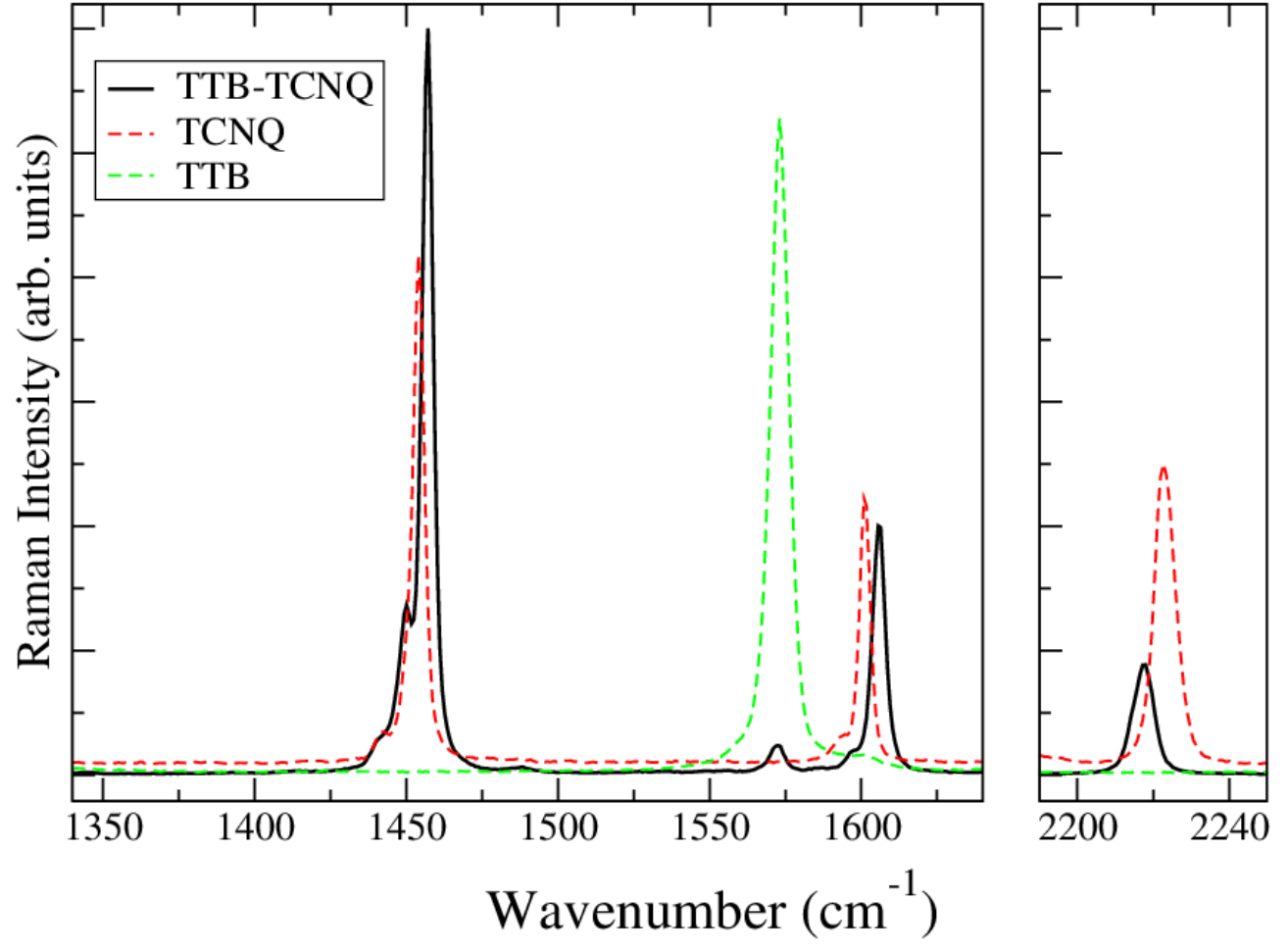

Fig. 5. Raman spectrum of TTB-TCNQ (black line) in the spectral region of the frequency sensitive modes. The Raman spectra of neutral TCNQ (dashed red line) and of TTB (dashed green line) are also shown for comparison. Exciting line: $676 \mathrm{~nm}$.

The absorption spectrum in the leftmost panel of Fig. 4, with polarization along the stack axis, locates the CT transition of TTB-TCNQ. We explicitly remark that we had problems in recording this part of the spectrum, since the samples are relatively thick, of prismatic shape, and quite often geminated. We attempted to obtain reflectance spectra, but the reflectivity is low and distorted by multiple internal reflections. The absorption spectrum of Fig. 4 clearly saturates in this spectral region, and the absorbance cannot be compared with that of the other two panels in the Figure. Neverthless, the spectrum allows to estimate the frequency of the CT transition at about $7000 \mathrm{~cm}^{-1}$, or $0.87 \mathrm{eV}$. From the frequency of the CT transition, the above guess of $\rho$, and the model calculation of Ref. [28], we can estimate the value of the DA hopping or CT integral, $t$, as $0.16 \mathrm{eV}$.

Some of us have recently developed a method to estimate relevant model parameters of mixed stack CT crystals via density-functional theory calculations [20]. Briefly, one calculates the singlet and triplet

lowest energy states for an isolated DA pair by keeping the geometry fixed at the one found from the X-ray analysis of the crystal. From these results one can evaluate $t$ and $z$, half the energy required to form an ionic pair. Furthermore, from the calculation of the charge distribution of both D and A in the neutral and fully ionized state, one calculates $V$, the DA intermolecular Coulomb potential, and $M$, the Madelung energy. In the present case, we find $t=0.21 \mathrm{eV}$, in satisfactory agreement with the above 
experimental estimate, and $z=0.48 \mathrm{eV}$. Moreover, $V=-1.92 \mathrm{eV}$ and $M=-0.97 \mathrm{eV}$. If we compare

these values with those obtained for the 11 mixed stack CT crystals considered in Ref. [20], we see that $t$ is on the lower side of the spanned values, and $z$ reflects the TTB ionization potential. The Madeleng energy, on the other hand, is among the smallest in the series, a fact that explains why TTB-TCNQ charge transfer in the crystal is well on the neutral side.

\section{Conclusions}

In summary, we have characterized an old, little studied electron donor and its mixed stack CT crystal with TCNQ. Whereas its ionization potential is not dramatically higher than that of TTF, the degree of CT in TTB-TCNQ is low. We think that this fact is due to the molecular arrangement of the molecules within the crystal, which is a compromise between the overlap between the frontier orbitals of the two molecules and the steric hindrance. TTB and TCNQ long axes are almost perpendicular one another, and this makes the 3D packing and density rather unfavorable, hence a Madelung energy lower than in other CT crystals. At the same time the CT integral is rather small, but the gap, as estimated by the optical gap (rightmost panel of Fig. 4), is below $1 \mathrm{eV}$ : According to the experiments by Tsutsumi et al. [29], TTB-TCNQ may exhibit good photocarrier generation and transport. In any case, it is worthwhile to investigate the CT complexes of TTB with other electron acceptors which might provide a better overlap and 3D packing.

\section{Acknowledgements}

We thank the late Prof. Daniel J. Sandman for his help with the synthesis. We acknowledge the support of the EU with ERC StG 2012-306826 e-GAMES and of the MINECO of the Spanish Government (CTQ2016- 80030-R and SEV-2015-0496).

\section{REFERENCES}

[1] L. Zhu, Y.Yi, Y. Li, E.-G. Kim, V.Coropceanu, J.-L. Brédas, Prediction of Remarkable Ambipolar Charge-Transport Characteristics in Organic Mixed-Stack Charge-Transfer Crystals, J. Am. Chem. Soc. 134 (2012) 2340-2347. doi:10.1021/ja210284s.

[2] S. Horiuchi, K. Kobayashi, R. Kumai, S. Ishibashi, Ionic versus Electronic Ferroelectricity in Donor-Acceptor Molecular Sequences, Chem. Lett. 43 (2014) 26-35. doi:10.1246/cl.130840.

[3] D. Vermeulen, L.Y. Zhu, K.P. Goetz, P. Hu, H. Jiang, C.S. Day, O.D. Jurchescu, V. Coropceanu, C. Kloc, L.E. McNeil, Charge Transport Properties of Perylene?TCNQ Crystals: The Effect of Stoichiometry, J. Phys. Chem. C. 118 (2014) 24688-24696. doi:10.1021/jp508520x. 
[4] J. Tsutsumi, S. Matsuoka, S. Inoue, H. Minemawari, T. Yamada, T. Hasegawa, N-type field-effect transistors based on layered crystalline donor-acceptor semiconductors with dialkylated benzothienobenzothiophenes as electron donors, J Mater Chem C. 3 (2015) 1976-1981. doi:10.1039/C4TC02481H.

[5] P. Hu, K. Du, F. Wei, H. Jiang, C. Kloc, Crystal Growth, HOMO-LUMO Engineering, and Charge Transfer Degree in Perylene-F ${ }_{x}$ TCNQ $(x=1,2,4)$ Organic Charge Transfer Binary Compounds, Cryst. Growth Des. 16 (2016) 3019-3027. doi:10.1021/acs.cgd.5b01675.

[6] T. Salzillo, M. Masino, G. Kociok-Kohn, D. Di Nuzzo, E. Venuti, R.G. Della Valle, D. Vanossi, C. Fontanesi, A. Girlando, A. Brillante, E. Da Como, Structure, Stoichiometry, and Charge Transfer in Cocrystals of Perylene with TCNQ-Fx., Cryst. Growth Des. 16 (2016) 3028-3036. doi:10.1021/acs.cgd.5b01663.

[7] A. Morherr, S. Witt, A. Chernenkaya, J.-P. Bäcker, G. Schönhense, M. Bolte, C. Krellner, Crystal growth of new charge-transfer salts based on $\pi$-conjugated donor molecules, Phys. B Condens. Matter. 496 (2016) 98-105. doi:10.1016/j.physb.2016.05.023.

[8] A. Chernenkaya, A. Morherr, S. Backes, W. Popp, S. Witt, X. Kozina, S.A. Nepijko, M. Bolte, K. Medjanik, G. Öhrwall, C. Krellner, M. Baumgarten, H.J. Elmers, G. Schönhense, H.O. Jeschke, R. Valentí, Microscopic origin of the charge transfer in single crystals based on thiophene derivatives: A combined NEXAFS and density functional theory approach, J. Chem. Phys. 145 (2016) 034702. doi:10.1063/1.4958659.

[9] K.P. Goetz, J. Tsutsumi, S. Pookpanratana, J. Chen, N.S. Corbin, R.K. Behera, V. Coropceanu, C.A. Richter, C.A. Hacker, T. Hasegawa, O.D. Jurchescu, Polymorphism in the 1:1 Charge-Transfer Complex DBTTF-TCNQ and Its Effects on Optical and Electronic Properties, Adv. Electron. Mater. (2016) 1600203. doi:10.1002/aelm.201600203.

[10] A. Girlando, Comment on Polymorphism in the 1:1 Charge-Transfer Complex DBTT-TCNQ and Its Effects on Optical and Electronic Properties, Adv. Electron. Mater. 3 (2017) 1600437. doi:10.1002/aelm.201600437.

[11] D.J. Sandman, G.D. Zoski, W.A. Burke, G.P. Hamill, G.P. Ceasar, A.D. Baker, Synthesis, electronic structure, and complex formation of simple 1,1,4,4-tetrathiabutadienes, J. Chem. Soc. Chem. Commun. (1981) 829. doi:10.1039/c39810000829.

[12] APEX2, SAINT and SADABS, Bruker AXS Inc., Madison, Wisconsin, USA., n.d.

[13] A. Altomare, M. C. Burla, M. Camalli, G. Cascarano C. Giacovazzo, A. Guagliardi, A. G. G. Moliterni, G. Polidori, R. Spagna, SIR97: a new tool for crystal structure determination and refinement, J. Appl. Crystallogr. 32 (1999) 115-119. doi: 10.1107/S0021889898007717.

[14] G.M. Sheldrick, Crystal structure refinement with SHELXL, Acta Crystallogr. Sect. C Struct. Chem. 71 (2015) 3-8. doi:10.1107/S2053229614024218.

[15] C. F. Macrae, I. J. Bruno, J. A. Chisholm, P. R. Edgington, P. McCabe, E. Pidcock, L. RodriguezMonge, R. Taylor, J. van de Streek and P. A. Wood, Mercury CSD 2.0 - New features for the visualization and investigation of crystal structures, J. Appl. Cryst. 41 (2008) 466-470. doi: 10.1107/S0021889807067908.

[16] Jmol: an open-source Java viewer for chemical structures in 3D, n.d. http://www.jmol.org/.

[17] M.S. Gordon, M.W. Schmidt, Advances in electronic structure theory: GAMESS a decade later, in: C.E. Dykstra, G. Frenking, K.S. Kim, G.E. Scuseria (Eds.), Theory Appl. Comput. Chem. First Forty Years, Elsevier, Amsterdam, 2005: pp. 1167-1189.

[18] V. Barone, G. Festa, A. Grandi, N. Rega, N. Sanna, Accurate vibrational spectra of large molecules by density functional computations beyond the harmonic approximation: the case of uracil and 2-thiouracil, Chem. Phys. Lett. 388 (2004) 279-283. doi:10.1016/j.cplett.2004.03.024.

[19] G. D’Avino, M.J. Verstraete, Are Hydrogen-Bonded Charge Transfer Crystals Room Temperature Ferroelectrics?, Phys. Rev. Lett. 113 (2014) 237602. doi:10.1103/PhysRevLett.113.237602.

[20] F. Delchiaro, A. Girlando, A. Painelli, A. Bandyopadhyay, S.K. Pati, G. D’Avino, Towards firstprinciples prediction of valence instabilities in mixed stack charge-transfer crystals, Phys. Rev. B. 95 (2017) 155125. doi:10.1103/PhysRevB.95.155125.

[21] M.J. Frisch, et al., Gaussian 09, Gaussian, Inc. Wallingford CT 2016, 2016. 
[22] G. D’Avino, L. Muccioli, C. Zannoni, D. Beljonne, Z.G. Soos, Electronic Polarization in Organic Crystals: A Comparative Study of Induced Dipoles and Intramolecular Charge Redistribution Schemes, J. Chem. Theory Comput. 10 (2014) 4959-4971. doi:10.1021/ct500618w.

[23] P. Ranzieri, M. Masino, A. Girlando, Charge-Sensitive Vibrations in $p$-Chloranil: The Strange Case of the CC Antisymmetric Stretching, J. Phys. Chem. B. 111 (2007) 12844-12848. doi:10.1021/jp075510v.

[24] A. Girlando, Charge Sensitive Vibrations and Electron-Molecular Vibration Coupling in Bis(ethylenedithio)-tetrathiafulvalene (BEDT-TTF), J. Phys. Chem. C. 115 (2011) 19371-19378. doi:10.1021/jp206171r.

[25] A. Girlando, R. Bozio, C. Pecile, J.B. Torrance, Discovery of vibronic effects in the Raman spectra of mixed-stack charge-transfer crystals, Phys. Rev. B. 26 (1982) 2306-2309. doi:10.1103/PhysRevB.26.2306.

[26] M. Meneghetti, C. Pecile, Charge-transfer organic crystals: Molecular vibrations and spectroscopic effects of electron-molecular vibration coupling of the strong electron acceptor TCNQF 4, J. Chem. Phys. 84 (1986) 4149-4162. doi:10.1063/1.450086.

[27] R. Bozio, I. Zanon, A. Girlando, C. Pecile, Influence of the intermolecular charge transfer interaction on the solution and solid state infrared spectra of 7,7,8,8-tetracyanoquinodimethane (TCNQ) alkaline salts, J. Chem. Soc. Faraday Trans. 2. 74 (1978) 235. doi:10.1039/f29787400235.

[28] A. Painelli, A. Girlando, Mixed regular stack charge-transfer crystals: Fundamental

microscopic parameters from optical spectra, J. Chem. Phys. 87 (1987) 17051711. doi:10.1063/1.453236.

[29] J. Tsutsumi, H. Matsui, T. Yamada, R. Kumai, T. Hasegawa, Generation and Diffusion of Photocarriers in Molecular Donor-Acceptor Systems: Dependence on Charge-Transfer Gap Energy, J. Phys. Chem. C. 116 (2012) 23957-23964. doi:10.1021/jp308720d. 


\title{
Supplemental Material for the paper:
}

Extensive study of the electron donor 1,1,4,4-

tetrathiabutadiene (TTB) and of its charge transfer crystal with

TCNQ

\author{
Nicola Castagnetti, Alberto Girlando, Matteo Masino, and Corrado Rizzoli \\ Dipartimento di Scienze Chimiche, della Vita e della Sostenibilità Ambientale, \\ and INSTM-UdR Parma, Università di Parma, \\ Parco Area delle Scienze 17/a, I-43124 Parma, Italy
}

M. R. Ajayakumar, Marta Mas-Torrent, and Concepció Rovira

Department of Molecular Nanoscience and Organic Materials,

Institut de Ciència de Materials de Barcelona (ICMAB-CSIC)

and Networking Research Center on Bioengineering, Biomaterials

and Nanomedicine (CIBER-BBN), ES-08193 Bellaterra, Spain

\section{Synthesis of TTB}
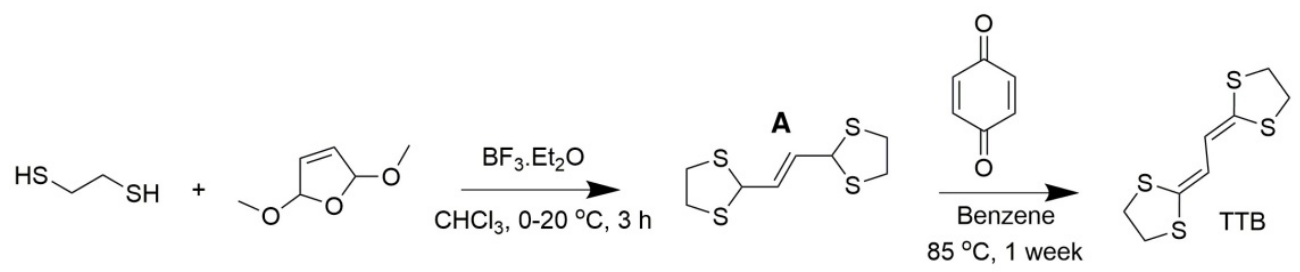

Ethane-1,2-dithiol ( $8 \mathrm{~mL}, 93.5 \mathrm{mmol}$ ) and 2,5-dimethoxy-2,5-dihydrofuran (5.2 mL, $41.6 \mathrm{mmol}$ ) in $60 \mathrm{~mL}$ of dry $\mathrm{CH}_{2} \mathrm{Cl}_{2}$ were stirred at $0 \mathrm{C}$ in a 2-neck round bottom flask under $\mathrm{Ar} .10 \mathrm{~mL}$ of $\mathrm{BF}_{3} \cdot \mathrm{Et}_{2} \mathrm{O}$ was injected very slowly by using a syringe with uniform stirring and the mixture was allowed to warm to $20 \mathrm{C}$ over a period of $3 \mathrm{~h}$. The precipitate obtained was filtered and washed with diethyl ether. It was further purified with flash column chromatography (silica gel, CH2Cl2:hexane 3:5, v/v) to afford compound A as white precipitate (2.4 g, 25\%). ${ }^{1} \mathrm{HNMR}\left(250 \mathrm{MHz}, \mathrm{CDCl}_{3}\right): 5.69(2 \mathrm{H}$, dd), $5.02(2 \mathrm{H}, \mathrm{dd})$,

$3.27(8 \mathrm{H}, \mathrm{m})$.

Compound A (3.4 g, $14.4 \mathrm{mmol})$ and p-benzoquinone $(7.9 \mathrm{~g}, 73.1 \mathrm{mmol}$ ) were charged into a $250 \mathrm{~mL}$ Schlenk flask and degased with Ar. $160 \mathrm{~mL}$ of dry benzene was added and refluxed for 1 week. The dark brown solution obtained was diluted with 200 $\mathrm{mL}$ toluene and 


\begin{tabular}{|c|c|c|c|c|c|}
\hline \multirow[b]{2}{*}{ Label } & & \\
\hline & $\omega_{\text {exp. }}$ & $\omega_{\text {scal. }}$ & $\omega_{\text {unscal. }}$ & $\omega_{\text {scal. }}$ & $\omega_{\text {unscal. }}$ \\
\hline$a_{\mathrm{g}} v_{1}$ & & 3042.1 & 3164.3 & 3049.9 & 3172.3 \\
\hline$v_{2}$ & & 3020.3 & 3141.6 & 3041.7 & 3163.8 \\
\hline$v_{3}$ & 2992 & 3007.3 & 3128.0 & 3030.6 & 3152.3 \\
\hline$v_{4}$ & 2969 & 2962.3 & 3081.2 & 2978.0 & 3097.6 \\
\hline$v_{5}$ & 2921 & 2959.3 & 3078.1 & 2976.7 & 3096.2 \\
\hline$v_{6}$ & 1573 & 1591.6 & 1655.5 & 1541.6 & 1603.5 \\
\hline$v_{7}$ & & 1450.1 & 1508.4 & 1447.3 & 1505.5 \\
\hline$v_{8}$ & & 1438.8 & 1496.6 & 1434.8 & 1492.4 \\
\hline$v_{9}$ & 1318 & 1308.4 & 1360.9 & 1290.5 & 1342.3 \\
\hline$v_{10}$ & 1307 & 1283.8 & 1335.3 & 1289.5 & 1341.2 \\
\hline$v_{11}$ & 1245 & 1246.6 & 1296.6 & 1255.2 & 1305.6 \\
\hline$v_{12}$ & 1162 & 1149.1 & 1195.3 & 1223.4 & 1272.5 \\
\hline$v_{13}$ & 1143 & 1134.5 & 1180.0 & 1149.8 & 1195.9 \\
\hline$v_{14}$ & & 1095.1 & 1139.0 & 1099.9 & 1144.0 \\
\hline$v_{15}$ & 955 & 954.4 & 992.7 & 963.6 & 1002.3 \\
\hline$v_{16}$ & 931 & 948.4 & 986.5 & 959.7 & 998.2 \\
\hline$v_{17}$ & & 895.9 & 931.9 & 939.3 & 977.0 \\
\hline$v_{18}$ & & 845.8 & 879.7 & 839.7 & 873.4 \\
\hline$v_{19}$ & 822 & 811.1 & 843.6 & 828.8 & 862.1 \\
\hline$v_{20}$ & 684 & 663.5 & 690.1 & 676.4 & 703.6 \\
\hline$v_{21}$ & & 655.5 & 681.8 & 645.6 & 671.5 \\
\hline$v_{22}$ & & 643.5 & 669.3 & 638.0 & 663.6 \\
\hline$v_{23}$ & 480 & 466.5 & 485.2 & 463.4 & 482.0 \\
\hline$v_{24}$ & 463 & 443.2 & 461.0 & 457.7 & 476.0 \\
\hline$v_{25}$ & 444 & 427.9 & 445.1 & 431.4 & 448.8 \\
\hline$v_{26}$ & 299 & 291.7 & 303.4 & 244.7 & 254.5 \\
\hline$v_{27}$ & 229 & 242.4 & 252.1 & 219.2 & 228.0 \\
\hline$v_{28}$ & & 215.9 & 224.5 & 216.9 & 225.6 \\
\hline$v_{29}$ & 155 & 148.4 & 154.4 & 154.9 & 161.2 \\
\hline$v_{30}$ & & 44.7 & 46.5 & 89.5 & 93.1 \\
\hline
\end{tabular}

TABLE I: Experimental, scaled and unscaled calculated frequencies $\left(\omega, \mathrm{cm}^{-1}\right)$ of TTB and $\mathrm{TTB}^{+}\left(C_{\mathrm{i}}\right.$ symmetry). Calculated intensities $\left(\mathrm{D}^{2} \AA^{-2} \mathrm{amu}^{-1}\right)$ are reported only for $a_{\mathrm{u}}$ IR active modes. 
Table I

(contin

ued)

\begin{tabular}{|c|c|c|c|c|c|c|c|}
\hline \multirow[b]{2}{*}{ Label } & \multicolumn{4}{|c|}{ TTB TTB $^{+}$} & \multirow[b]{2}{*}{$\omega_{\text {scal. }}$} & \multirow[b]{2}{*}{$\omega_{\text {unscal. }}$} & \multirow[b]{2}{*}{ IR int. } \\
\hline & $\omega_{\text {exp. }}$ & $\omega_{\text {scal. }}$ & $\omega_{\text {unscal. }}$ & IR int. & & & \\
\hline$a_{\mathrm{u}} v_{31}$ & & 3051.1 & 3173.6 & 0.13979 & 3058.8 & 3181.7 & 0.06192 \\
\hline$v_{32}$ & 3003 & 3020.3 & 3141.6 & 0.54849 & 3041.7 & 3163.8 & 0.00219 \\
\hline$v_{33}$ & 2956 & 3007.3 & 3128.0 & 0.15225 & 3030.6 & 3152.3 & 0.00287 \\
\hline$v_{34}$ & 2920 & 2962.2 & 3081.1 & 1.62965 & 2978.1 & 3097.6 & 0.07510 \\
\hline$v_{35}$ & & 2959.4 & 3078.2 & 0.52615 & 2976.7 & 3096.2 & 0.04336 \\
\hline$v_{36}$ & 1524 & 1541.4 & 1603.2 & 4.34586 & 1447.2 & 1505.3 & 0.15533 \\
\hline$v_{37}$ & & 1450.0 & 1508.2 & 0.00306 & 1434.9 & 1492.5 & 0.55480 \\
\hline$v_{38}$ & 1415 & 1438.8 & 1496.5 & 0.24332 & 1380.1 & 1435.5 & 12.49079 \\
\hline$v_{39}$ & 1273 & 1282.3 & 1333.8 & 2.27983 & 1290.0 & 1341.8 & 0.13625 \\
\hline$v_{40}$ & & 1270.1 & 1321.1 & 0.81498 & 1285.5 & 1337.1 & 6.52965 \\
\hline$v_{41}$ & 1241 & 1242.3 & 1292.2 & 0.24307 & 1250.6 & 1300.8 & 0.56710 \\
\hline$v_{42}$ & 1147 & 1140.0 & 1185.7 & 0.08545 & 1150.4 & 1196.6 & 0.86802 \\
\hline$v_{43}$ & 1104 & 1095.4 & 1139.4 & 0.11832 & 1100.1 & 1144.2 & 0.05113 \\
\hline$v_{44}$ & 975 & 954.2 & 992.5 & 0.25573 & 963.5 & 1002.1 & 0.23410 \\
\hline$v_{45}$ & & 947.3 & 985.3 & 0.01378 & 950.3 & 988.4 & 0.19009 \\
\hline$v_{46}$ & 845 & 868.6 & 903.5 & 0.26638 & 909.5 & 946.0 & 2.53659 \\
\hline$v_{47}$ & 834 & 831.5 & 864.9 & 1.15079 & 886.6 & 922.2 & 0.76855 \\
\hline$v_{48}$ & & 818.6 & 851.5 & 0.58594 & 838.6 & 872.3 & 0.30940 \\
\hline$v_{49}$ & 682 & 663.6 & 690.3 & 0.03844 & 647.4 & 673.4 & 0.03556 \\
\hline$v_{50}$ & & 654.6 & 680.9 & 0.33384 & 639.9 & 665.6 & 1.01749 \\
\hline$v_{51}$ & & 550.9 & 573.0 & 0.85310 & 578.3 & 601.5 & 1.66569 \\
\hline$v_{52}$ & & 471.3 & 490.2 & 0.04412 & 513.4 & 534.0 & 0.04378 \\
\hline$v_{53}$ & & 449.2 & 467.2 & 0.13030 & 454.1 & 472.3 & 0.01359 \\
\hline$v_{54}$ & & 405.2 & 421.5 & 0.04778 & 426.2 & 443.3 & 0.18401 \\
\hline$v_{55}$ & & 401.6 & 417.7 & 0.30074 & 399.7 & 415.8 & 0.22625 \\
\hline$v_{56}$ & & 243.3 & 253.1 & 0.16083 & 217.9 & 226.7 & 0.20193 \\
\hline
\end{tabular}




\begin{tabular}{rrrrrrr}
$v_{57}$ & 94.6 & 98.4 & 0.03493 & 149.2 & 155.2 & 0.05791 \\
$v_{58}$ & 62.6 & 65.1 & 0.02683 & 65.4 & 68.0 & 0.01564 \\
$v_{59}$ & 34.7 & 36.1 & 0.16329 & 50.7 & 52.8 & 0.12389 \\
$v_{60}$ & 31.7 & 33.0 & 0.02761 & 34.4 & 35.8 & 0.00097 \\
\hline \hline
\end{tabular}

